\title{
Dysferlinopathy: Clinical aspects in India
}

\author{
Hidenori Hattori, Norihiro Suzuki \\ Department of Neurology, School of Medicine, Keio University, 35 Shinanomachi, Shinjuku-ku, Tokyo 160-8582, Japan
}

Mutations in the dysferlin gene on Chromosome 2p cause dysferlinopathies which include two distinct clinical entities, Miyoshi myopathy (MM) (OMIM no. 254130) and limb girdle muscular dystrophy 2B (LGMD2B) (OMIM no. 253601). They have been diagnosed as different diseases clinically depending on the affected muscles at the onset. In this issue of Neurology India, Nalini and Gayathri reported the differences in their clinical findings in India, ${ }^{[1]}$ while the histopathological characteristics of their muscle specimens were not any different. The authors have presented the largest study of dysferlinopathy in India which focused on clinical aspects, according to our knowledge .

MM and LGMD2B are autosomal recessive, slow progressive muscular dystrophies recognized by primary deficiency of dysferlin expression on their patients' muscles. The patients who have dysferlin deficiency are affected in the teens or early adulthood, and show dramatic elevation of serum creatine kinase (CK). ${ }^{[2]}$ Liu and colleagues discovered dysferlin, a skeletal muscle gene, which is found to be mutated in both MM and LGMD2B. ${ }^{[3]}$ The study employing dysferlin-null mice showed that the mice developed progressive muscular dystrophy and exhibited elevated serum CK like human dysferlinopathy. ${ }^{[4]}$ Dysferlin-deficient muscle fibers were defective in $\mathrm{Ca} 2+$-dependent sarcolemma resealing and it suggested the membrane repair system did not work during contraction-induced injuries. We reported that dantrolene, a well-known Ca2 + release inhibitor during muscle contraction, was effective for some Japanese MM siblings. ${ }^{[5]}$ Altogether these facts might support that both dysferlinopathies had the same pathological background.

Although both diseases have the same genetic and pathological background, they are distinguishable clinically. Basically, the calf muscles are affected at the initial stage in MM, but LGMD2B begins with proximal muscle weakness. The investigators focused on the clinical differences between MM and LGMD2B employing 28 Indian patients who had been diagnosed as dysferlinopathy by immunohistochemical analysis since $6.4 \pm 4.2$ years. They found that the occurrence of distal muscle weakness in upper limbs was seen specifically in MM patients. It was pretty interesting, because originally Miyoshi and colleagues focused on the lower limb weakness. ${ }^{[2]}$ Another Japanese group focused on the clinical differences between patients with and without G3370T or G3510A mutation in dysferlin gene, but in only MM. They found that the clinical onset was delayed in the patients with G3370T mutation and higher serum CK was seen in the patients with G3510A mutation. ${ }^{[6]}$ The same group has recently reported that a 53-year-old man with LGMD2B (homozygous G3370T) mutation had chorea in addition to the myopathy. This is perhaps explained by the expression of smaller brain isoform of dysferlin in putamen. ${ }^{[7]}$ These findings imply that the future genetic analysis might be able to provide new correlation between dysferlin mutation and clinical phenotype and much longer follow-up could uncover other interesting central nervous system-derived symptoms. As the authors noticed, they had to still exclude calpainopathy (LGMD2A) (OMIM no.253600) and caveolinopathy (LGMD1C) (OMIM no.607801). Three cases of LGMD2A showed a double defect of both calpain-3 and dysferlin in dystrophy muscles ${ }^{[8]}$ and dysferlin expression was patchy in LGMD1C muscles. ${ }^{[9]}$ Further genetic analysis or caveolin-3 immunostaining for LGMD1C might be helpful for differential diagnosis in the future. Nevertheless the present paper has outlined clinical details about both forms of dysfelinopathy in India preciously, and it will be further informative to uncover the geographical and ethnical differences of dysferlinopathy in the world.

\section{Acknowledgment}

We thank Dr. Tadayuki Ishihara of National Hakone Hospital, Kanagawa, Japan for his specialized advice as expert. This work was supported by a part of grant-in-aid to Hidenori Hattori 
for scientific research from the Ministry of Health, Labor and Welfare of Japan. There was no conflict of interest.

\section{References}

1. Nalini A, Gayathri N. Dysferlinopathy: A clinical and histopathological study of 28 patients from India. Neurol India 2008;58:379-385.

2. Miyoshi K, Kawai H, Iwasa M, Kusaka K, Nishino H. Autosomal recessive distal muscular dystrophy as a new type of progressive muscular dystrophy: Seventeen cases in eight families including an autopsied case. Brain 1986:109:31-54.

3. Liu J, Aoki M, Illa I, Wu C, Fardeau M, Angelini C, \& al. Dysferlin, a novel skeletal muscle gene, is mutated in Miyoshi myopathy and limb girdle muscular dystrophy. Nat Genet 1998;20:31-6.

4. Bansal D, Miyake K, Vogel SS, Groh S, Chen CC, Williamson R, \& al. Defective membrane repair in dysferlin-deficient muscular dystrophy. Nature 2003:423:168-72.

5. Hattori H, Nagata E, Oya Y, Takahashi T, Aoki M, Ito D, \&al. A novel compound heterozygous dysferlin mutation in Miyoshi myopathy siblings responding to dantrolene. Eur J Neurol 2007:14:1288-91.

6. Takahashi T, Aoki M, Tateyama M, Kondo E, Mizuno T, Onodera Y, \& al. Dysferlin mutations in Japanese Miyoshi myopathy: Relationship to phenotype. Neurology 2003;60:1799-804.

7. Takahashi T, Aoki M, Imai T, Yoshioka M, Konno H, Higano S, \& al. A case of dysferlinopathy presenting choreic movements. Mov Disord 2006;21:1513-5.

8. Fanin M, Pegoraro E, Matsuda-Asada C, Brown RH Jr, Angelini C. Calpain-3 and dysferlin protein screening in patients with limb-girdle dystrophy and myopathy. Neurology 2001;56:660-5.

9. Matsuda C, Hayashi YK, Ogawa M, Aoki M, Murayama K, Nishino I, \& al. The sarcolemmal proteins dysferlin and caveolin-3 interact in skeletal muscle. Hum Mol Genet 2001;10:1761-6.

Accepted on 25-03-2008

Source of Support: Grant-inaid to Hidenori Hattori for scientific research from the Ministry of Health, Labor and Welfare of Japan., Conflict of Interest: None declared. 